95355

\section{Potential Drug Therapy for Fragile X Tremor/Ataxia Syndrome}

Collis Brown, Tamaro Hudson and Sonya K.Sobrian

Howard University

ABSTRACT IMPACT: The ability to restore mitochondrial health in neurons derived from FXTAS patient-induced pluripotent stem cells by novel natural compounds is critically important to the management of patients experiencing this syndrome and other Fragile $\mathrm{X}$ associated disorders. OBJECTIVES/GOALS: The goal of this research is to assess the biological potency of MAM, NADA and MAM analogues' neuroprotective capacity with respect to mitochondrial damage, and antioxidant properties that can restore mitochondrial health in patients with FXTAS. METHODS/STUDY POPULATION: To establish mitochondrial dysfunction, normal human cell lines and human induced pluripotent cells will be exposed to multiple concentrations of glucose/ glucose oxidase (GluOx) at several time points to induce varying intensities of oxidative stress. The degrees of oxidative stress will be measured by apoptosis and mitochondrial reactive oxygen species (ROS) production. $\mathrm{N}$-arachidonoyldopamine (NADA), macamides (MAM) and its analogue compounds, effective against oxidative damage in mitochondria, will be used to rescue glucose oxidase induced oxidative damage in both cell lines. To test the ability of these drugs to restore mitochondrial health, cell viability and cellular superoxide production will be assessed by propidium iodide and the MitoSox fluorescence assay, respectively. RESULTS/ANTICIPATED RESULTS: We anticipate that GluOx at varying concentrations and time points will proportionally increase levels of apoptosis and mitochondrial ROS, reflective of mitochondrial damage, with the most severe dysfunction occurring at the maximum dose of $40 \mu \mathrm{M}$ and the longest duration of 72-hr exposure. Moreover, administration of NADA, MAM, and MAM analogues at seven concentrations, ranging from 10-8 to 10-5 $\mathrm{M}$ in half-log increments, will successfully treat the oxidative defects induced in the cell lines by decreasing apoptosis, and superoxide production, and increasing cell viability. DISCUSSION/ SIGNIFICANCE OF FINDINGS: This research allows for the development of an in vitro neuronal model of FXTAS, lends flexibility to testing therapeutics, and expands the discovery of mitochondrial biomedical markers for the syndrome. Data generated should inform mechanistic studies of the relationship between mitochondrial damage and FXTAS-induce neurodegeneration.

95923

TBI-on-a-chip: Linking physical impact to neurodegeneration by decrypting primary and secondary injury mechanisms

Edmond Rogers ${ }^{1}$, Timothy Beauclair ${ }^{1}$, Guenter Gross ${ }^{2}$ and Riyi Shi ${ }^{1}$ ${ }^{1}$ Purdue University and ${ }^{2}$ University of North Texas

ABSTRACT IMPACT: This unique approach has the capability to elucidate the pathological mechanisms underlying traumatic brain injuries and neurodegeneration, both separately and in concert, while simultaneously providing a semi-high throughput model for investigating potential pharmaceutical interventions: discoveries that would have major translational implications and a significant impact worldwide. OBJECTIVES/GOALS: We aim to improve our understanding of the mechanisms behind the development of neurodegenerative diseases by utilizing the link between traumatic brain injuries and demonstrated biomarkers with our innovative TBI on a chip model. With this tool, we hope to provide new pathological insights and explore potential pharmaceutical interventions. METHODS/STUDY POPULATION: E16 murine cortical networks were cultured onto reusable, optically transparent MEAs (fabricated in-house), and subjected to a clinically-relevant range (30-300g) of impact g-forces, utilizing our exciting new in vitro model of trauma (TBI on a chip) with real-time electrophysiological and morphological access. Impacts were systematically applied at varying intensities, repetitions, and time points, and fixed 24-hours post. Basic immunocytochemical techniques were used to investigate post-impact levels of acrolein, an established biomarker of both post-TBI oxidative stress and neurodegeneration (ND), and compared to procedurally and age-matched non-impact control networks. In addition, several other TBI/ND biomarker investigations are in progress ( $\beta A 42$, $\alpha$-synuclein, and phosphorylated tau). RESULTS/ANTICIPATED RESULTS: Impact experiments revealed significant, force-dependent increases of acrolein (acrolein-lysine adducts) at 24hrs post impact, indicative of impact-linked neuronal degeneration. These changes were amplified by the following manipulations: increasing g-force exposure (30-250 g); the rapid (4-6 sec interval) application of multiple impacts (1, 3, 5 and 10x); and exposure to $40 \mathrm{mM} \mathrm{EtOH} \mathrm{(10} \mathrm{min}$ duration immediately following impact). Further, we demonstrate the enhancement of injury recovery as a function of: increasing time intervals between repeated hits; Hydralazine exposure. In addition, conditioned media from maximally-impacted cultures can cause acrolein elevation when introduced to non-impact, control networks, indicating acrolein's role as a diffusive-factor in post-TBI secondary injuries. DISCUSSION/SIGNIFICANCE OF FINDINGS: This novel approach provides unprecedented resolution, and is improving our understanding of the pathological mechanisms underlying both TBI and ND. Combined with our established in vivo models of trauma and computer modeling, we hope to better guide our translational laboratory endeavors and help improve clinical diagnoses and treatments.

\section{Clinical Epidemiology}

76352

Investigating the Association Between Intracerebral Hemorrhage (ICH) and Long-Term Encephalomalacia and Cortical Atrophy

Arjun Adapa ${ }^{1}$, Siri Sahib Khalsa ${ }^{2}$, Joseph Linzey ${ }^{2}$ and

Aditya Pandey ${ }^{2}$

${ }^{1}$ University of Michigan Medical School and ${ }^{2}$ University of Michigan Department of Neurosurgery

ABSTRACT IMPACT: Investigating the relationship between ICH volume at presentation and cortical atrophy over time may help to explain the phenomenon of cognitive impairment after ICH. OBJECTIVES/GOALS: Non-traumatic intracerebral hemorrhage (ICH) is the most common type of hemorrhagic stroke. ICH causes both mechanical as well as oxidative injury leading to neurologic deterioration. However, the relationship between ICH volume and brain atrophy is not fully understood. To that end, we aimed to investigate this relationship. METHODS/STUDY POPULATION: A retrospective cohort of adult ICH patients over a 10-year study period were studied. Demographic data, ICH cause, location, 
laterality, and treatment, as well as other data, were collected. DICOM files of CT or MRI at presentation and all subsequent follow-up imaging up to 5 -years post-ICH were obtained. Using a novel semi-automated image segmentation tool developed in MATLAB by our group, ICH volumes were segmented. Using T1w MRI data, ipsiand contralateral brain volumes were segmented using NeuroQuant, a fully automated software for MRI volumetric processing, for both initial and follow-up MRIs. Ipsilateral and global cerebral volume changes over time were calculated using initial and follow-up MRI images. Spearman rank correlation between volume changes and ICH volumes were calculated for each patient. RESULTS/ ANTICIPATED RESULTS: 75 spontaneous ICH patients with adequate imaging follow-up and both early and follow-up MRI comparisons were considered. Of these, 14 patients had MRIs adequate for segmentation by NeuroQuant. There was a positive correlation $\left(\mathrm{R}^{\wedge} 2=0.72, \mathrm{p}=0.03\right)$ between $\mathrm{ABC} / 2$, the traditional $\mathrm{ICH}$ volume measuring approach, and our semi-automatic segmentation tool, with $\mathrm{ABC} / 2$ tending to overestimate $\mathrm{ICH}$ volume. There was an average of $-6.51 \%$ of total brain volume loss with respect to initial brain volume at follow-up. There was a negative correlation between ICH volume and both global (Spearman rank correlation coefficient $(\mathrm{R})=-0.714, \mathrm{p}=0.004))$ and local atrophy $(\mathrm{R}=-0.785, \mathrm{p}=0.0009)$, meaning that as ICH volume increases, there is greater brain volume loss. DISCUSSION/SIGNIFICANCE OF FINDINGS: Greater ICH volume is associated with greater brain volume loss both ipsilaterally, reflected as encephalomalacia, and globally. These findings are important as encephalomalacia can result in focal neurologic deficit and other neurological symptoms over time, while global brain atrophy is associated with dementia and cognitive decline.

\section{Clinical Trial}

79696

Reversible DNA Hypermethylation of the Interleukin-15

Promoter Induces IL-15 Expression, Drives the Pathogenesis of T-Cell Large Granular Lymphocytic Leukemia (T-LGLL) and Provides a Therapeutic Approach Using 5-Azacitidine

Jonathan E Brammer ${ }^{1}$, Anjali Mishra², Amy Boles², Anthony

Mansour $^{3}$, Monique Mathe-Allainmat ${ }^{4}$, Agnes Quemener ${ }^{5}$ and Pierluigi Porcu ${ }^{2}$

${ }^{1}$ The Ohio State University James Cancer Center, ${ }^{2}$ Thomas Jefferson University, ${ }^{3}$ City of Hope Cancer Center, ${ }^{4}$ Universite de Nantes and ${ }^{5}$ Universite de Nantes Erwan Mortier, Universite de Nantes

ABSTRACT IMPACT: This work describes, for the first time, the methylome in patients with T-LGLL, focusing on the IL-15 promoter, and clearly demonstrates that 5-azacytidine decreases IL-15 production leading to T-LGLL cell death. These results form the basis a translational clinical trial in T-LGLL that will begin accrual in 2021 OBJECTIVES/GOALS: T-LGLL is an incurable leukemia with few treatment options driven by overexpression of IL-15. Our objective is to characterize the methylation status of the IL-15 promoter in TLGLL patients and evaluate the potential use of 5-azacytidine (5-aza) in a translational trial by studying the effect of 5-aza in vitro on IL-15 levels, and the IL-15 promoter. METHODS/STUDY POPULATION: We sorted T-LGLL patient $(\mathrm{n}=3)$ and normal donor
(ND) samples $(n=3)$ for $\mathrm{CD} 3+/ \mathrm{CD} 8+/ \mathrm{CD} 5-/$ dim for T-LGLL immunophenotype. We analyzed DNA methylation and gene expression profiling using reduced representation bisulfite and RNA sequencing and determined differential methylation and gene expression using 1-way ANOVA analysis. To determine the functional significance of differential methylation, we evaluated MOTN-1 T-LGLL cell viability in vitro with 5-aza at increasing concentrations. Next, we evaluated IL-15 gene expression in MOTN-1 cells treated with 5-Aza versus MOTN-1 with control using western immunoblot. Finally, we exposed MOTN-1 cells to a novel IL-15 inhibitor, IBI-15, and compared cell viability against MOTN-1 cells exposed to an inactive control. RESULTS/ANTICIPATED RESULTS: There was significant differential methylation ( $\mathrm{P}=$ 0.0178 ) and expression ( $\mathrm{P}=0.0059)$ in T-LGLL patients vs ND. These data revealed significant differential hypermethylation of gene promoters, including an increase in DNA methylation of the IL-15 promoter in T-LGLL cells vs ND. In MOTN-1 cells treated in vitro with 5-Aza at 24 and 48 hours, a dose-dependent decrease in the viability of T-LGLL cells was observed, from $100 \%$ to $49.5 \%$, $\mathrm{p}=0.037$. Further, a marked decrease in IL-15 expression was observed at all concentrations of 5-aza compared to control $(\mathrm{p}=0.0001)$. Finally, a decrease in cell viability was observed utilizing the IL-15 inhibitor IBI- 15 vs control. These results confirm that 5-aza leads to decreased transcription of the IL-15 gene, possibly due to hypomethylation of the IL-15 promoter. DISCUSSION/ SIGNIFICANCE OF FINDINGS: Hypermethylation of the IL-15 promoter and subsequent increase in IL-15 is critical to the pathogenesis of T-LGLL. Inhibition of the IL- 15 promoter by 5 -aza leads to down-regulation of the IL-15 gene transcript, which is sufficient to induce T-LGLL cell death. Based on these results, a phase I trial will be conducted using CC-486 (oral 5-Aza) in T-LGLL.

\section{Data Science/Biostatistics/Informatics}

\section{4}

\section{Electrical stimulation of hippocampus and amygdala produces multiple distinct responses in human ventral temporal cortex}

Harvey Huang, Nicholas M. Gregg, Gabriela Ojeda, Benjamin H. Brinkmann, Brian N. Lundstrom, Gregory A. Worrell, Kai J. Miller and Dora Hermes

Mayo Clinic

ABSTRACT IMPACT: This study characterizes interactions between human limbic circuitry and ventral temporal cortex using single pulse electrical stimulation, which may inform emerging stimulation therapies for epilepsy. OBJECTIVES/GOALS: The goal of electrical brain stimulation treatment is to modulate brain network function. However, stimulation inputs to different brain sites alter the network in a variety of ways. This study examines that variability by characterizing responses in a target region while stimulating multiple other brain sites. METHODS/STUDY POPULATION: We measured voltages in intracranial EEG in 6 patients who had electrodes implanted for epilepsy monitoring. We stimulated pairs of electrodes at multiple sites in the brain with a single pulse every 5 to $7 \mathrm{~s}$ and measured the resulting corticocortical evoked potential (CCEP) responses in the ventral temporal cortex (VTC). Using a novel clustering method, we uncovered sets of distinct canonical 